\title{
Carnets
}

Revue électronique d'études françaises de l'APEF

Première Série - 4 | 2012

(Res)sources de l'extravagance

\section{Figures du lecteur extravagant au XVIIe siècle : de la satire des fictions fabuleuses à l'éloge de l'imagination créatrice}

\section{Françoise Poulet}

\section{(2) OpenEdition}

Journals

Édition électronique

URL : http://journals.openedition.org/carnets/6530

DOI : $10.4000 /$ carnets. 6530

ISSN : 1646-7698

Éditeur

APEF

Édition imprimée

Date de publication : 1 janvier 2012

Pagination : 11-30

Référence électronique

Françoise Poulet, «Figures du lecteur extravagant au XVIle siècle : de la satire des fictions fabuleuses à l'éloge de l'imagination créatrice », Carnets [En ligne], Première Série - 4 | 2012, mis en ligne le 20 juin 2018, consulté le 01 mai 2019. URL : http://journals.openedition.org/carnets/6530 ; DOI : 10.4000/ carnets. 6530

\section{(2) $(1) \Theta$}

Carnets est mis à disposition selon les termes de la licence Creative Commons - Atribution - Pas d'utilisation commerciale 4.0 International. 


\title{
FIGURES DU LECTEUR EXTRAVAGANT AU XVII SIECLE \\ De la satire des fictions fabuleuses à l'éloge de l'imagination créatrice
}

FRANÇOISE POULET

Université de Poitiers

franc.poulet@gmail.com

\begin{abstract}
Résumé
La parution de Don Quichotte en Espagne est suivie d'une vaste série de traductions, d'adaptations et d'imitations au sein de la littérature française. On voit même apparaître une lignée de lecteurs extravagants dans le genre de l'histoire comique, à partir de la publication du Berger extravagant de Sorel, en 1627-1628. Lysis, mais aussi Don Clarazel dans Le Chevalier hypocondriaque de Du Verdier, puis Juliette d'Arviane dans La Fausse Clélie de Subligny, basculent dans la folie pour avoir abusé de lectures romanesques recourant sans limites aux ressources de l'imaginaire. Au travers de ces personnages, il s'agit de mettre en garde contre un type de lecture qui s'abandonne sans recul aux dangers de la fable. Mais les œuvres que nous nous proposons d'étudier fêtent également avec gaieté les plaisirs de l'imagination. Aussi le personnage extravagant permet-il de faire l'éloge d'un mode de lecture prudent et avisé, au sein de romans à l'inventivité maîtrisée.
\end{abstract}

\begin{abstract}
The publication of Don Quixote in Spain is followed by a set of translations, adaptations and imitations in French literature. We can even see the emergence of many "extravagant readers" in comic novels, starting from the publication of Le Berger extravagant by Sorel in 1627-1628. The character of Lysis, that of Don Clarazel in Le Chevalier hypocondriaque by Du Verdier, and then of Juliette d'Arviane in La Fausse Clélie by Subligny, all become insane for having read too many novels using imaginative resources without limits. With the creation of such characters, the intent is to warn the reader against a certain type of reading which would yield completely to the dangers of Fiction. However, the comic novels I would like to study here also joyfully celebrate the pleasures of imagination. This is why the presence of the extravagant character involves a careful and informed type of reading in novels where inventiveness is controlled.
\end{abstract}

Mots-clés: extravagance, lecteur, roman, fabuleux, imagination

Keywords: extravagancy, reader, novel, fantasy, imagination 
"[...] pardonnez moy si je dis que vous extravaguez merveilleusement, cet Amadis de Gaule ny cet Agesilan de Colchos desquels vous parlez n'ont jamais esté qu'en peinture" (Du Verdier, 1632: 292): contrairement à ce que l'on pourrait croire de prime abord, ce n'est pas à Don Quichotte que le personnage qui s'exprime ici - il s'agit d'un capucin - s'adresse, mais à l'un de ses avatars et héritiers français des premières décennies du XVII ${ }^{\mathrm{e}}$ siècle, Don Clarazel, héros du Chevalier hypocondriaque de Du Verdier. En effet, peu après sa parution en deux volumes, respectivement en 1605 et 1615, l'œuvre de Cervantès rencontre un succès important et durable chez ses lecteurs français, ce dont témoignent ses traductions précoces: César Oudin en publie la première partie dès 1614, tandis que François de Rosset en traduit la seconde en 1618. Les imitations et adaptations de ce récit sont immédiates et touchent tous les genres: investissant tout d'abord le ballet de cour, Don Quichotte fournit par la suite la matière de pièces de théâtre et de romans. En 1630, Pichou donne par exemple une tragi-comédie intitulée Les Folies de Cardenio; Guérin de Bouscal offre quant à lui au public une trilogie comique intitulée Dom Quixote de la Manche (1639), Dom Quichot de la Manche. Seconde partie (1640) et Le Gouvernement de Sanche Pansa (1642). Charles Sorel, lorsqu'il publie Le Berger extravagant en 1627-1628, est le premier auteur à s'inspirer de l'œuvre espagnole dans le genre romanesque. À sa suite, une vogue de lecteurs devenus fous envahit la littérature, et plus particulièrement l'histoire comique ${ }^{1}$ : outre Le Chevalier hypocondriaque déjà cité, on peut mentionner Le Dom Quixote Gascon attribué au comte de Cramail (1630), La Fausse Clélie de Subligny (1671), et même, au cours du siècle suivant, L'Histoire des imaginations extravagantes de monsieur Oufle de Laurent Bordelon (1710), La Voiture embourbée (1714) et Pharsamon ou les Nouvelles Folies Romanesques (1737) de Marivaux ${ }^{2}$. Ces différentes œuvres mettent toutes en scène des personnages qui, comme Don Quichotte, conçoivent le monde qui les entoure comme une duplication de l'univers fabuleux qu'ils ont arpenté au cours de leurs lectures. Toutefois, au fil du siècle, la cible romanesque visée évolue: si Du Verdier conserve l'attaque portée par Cervantès contre les romans de chevalerie, ce sont les fictions pastorales qui conduisent le jeune bourgeois Louis à emprunter le surnom de Lysis et à se faire berger, dans Le Berger extravagant, tandis que I'héroïne de Subligny se prend pour une nouvelle Clélie, tout droit sortie du célèbre roman de Mlle de Scudéry.

Plutôt que d'être désignés comme fous, ces lecteurs sont généralement qualifiés par un même adjectif: celui d'extravagant. À la suite du Berger de Sorel, une lignée de héros extravagants se constitue, comme l'indique Du Verdier dans l'avis "Au lecteur" du Chevalier:

\footnotetext{
1 Sur ce genre romanesque qui apparaît à partir du début du XVII siècle en marge des grands romans sentimentaux, pastoraux, puis héroïques, voir Jean Serroy, Roman et réalité: les histoires comiques au XVII siècle (1981).

${ }^{2}$ II serait bien entendu trop long de citer l'intégralité des œuvres françaises plus ou moins directement inspirées du Quichotte: voir à ce titre la bibliographie présentée par Maurice Bardon (Bardon, 1931: 852 ss), de même que les ouvrages de Jean-Paul Sermain (Sermain, 1999 et 2002).
} 
[...] De sorte qu'ayant resolu de publier les principaux traits de follie qu'il à faits \& qui sont venus à ma cognoissance j'aurois fait cogoistre mon livre par le nom DU Chevalier Extravagant, si cet epithete n'eust esté donné avec jugement à un BERGER dont les imaginations n'estoient gueres plus sages que les pensees ou les fantaisies de nostre guerrier. L'autheur de ce fameux Roman, m'ayant donc preveu dans la rencontre de ce tiltre qui seroit propre à mon subjet, j'appelleray le mien LE CHEVALIER HYPOCONDRIAQUE \& supplieray tout d'un mesme temps ceux qui prendront plaisir à voir ses boutades de considerer qu'elles sont fort differentes de celles du Berger qui la preceddé \& par consequent de croire que je n'ay rien voulu emprunter de ses mouvemens non plus que de l'invention du chevalier Espagnol lequel à commencé de monstrer la foiblesse de son cerveau par des impressions fantasticques \& des actions ridicules (Du Verdier, 1632: n. p.).

Si l'auteur du Chevalier hypocondriaque choisit une autre épithète en vue de distinguer son œuvre de celle de Sorel, il montre que son héros s'inscrit au sein d'une filiation dont il a pleinement conscience. Même si les aventures de Clarazel ne sont pas présentées comme une pure décalque de celles des personnages qui l'ont précédé, le principe reste le même: le chevalier quitte les îles Baléares pour se faire chevalier errant, puis parcourt la Bourgogne et le Lyonnais afin de vivre des aventures héroïques et amoureuses semblables à celles qui sont relatées dans les romans d'Amadis. Plusieurs décennies plus tard, lorsque Subligny compose sa Fausse Clélie, l'emploi récurrent du terme extravagance prouve une fois encore que l'héroïne de cette "histoire françoise, galante et comique" est conçue comme la descendante de Lysis et de Clarazel, mais aussi, à travers eux, de Don Quichotte: Juliette d'Arviane traverse de longues phases de lucidité, mais, dès que quelqu'un fait allusion à l'histoire romaine devant elle, elle "retomb[e] peu à peu dans son extravagance" (Subligny, 1971: 159).

Contrairement au fou, dont l'étymologie (follis) renvoie au soufflet, au ballon gonflé d'air, l'extravagant est loin d'avoir la tête vide: plutôt que d'abdiquer toute activité réflexive, il fait fonctionner avec excès les capacités de son jugement, en leur donnant pour appui, de surcroît, une faculté imaginative perturbée. Conformément au double sens spatial et psychologique que revêt le verbe vagor en latin classique, l'extra-vagant est celui dont la pensée s'écarte des chemins battus, qui emprunte une autre voie que celle qui est communément suivie. Cet écart peut alors le conduire à s'égarer dans les méandres d'une pensée qui tourne à vide; mais il peut aussi le mener vers les pentes capricieuses de l'imaginaire le plus inventif. C'est ainsi qu'une double approche ambivalente de ce personnage se trouve adoptée dans les histoires comiques que nous avons mentionnées et que nous prendrons ici pour objets d'étude: d'une part, le lecteur extravagant sert de relais à 
une critique métalittéraire formulée à l'encontre d'une certaine conception de la fiction et du genre romanesque. Mais la notion d'extravagance est loin de donner lieu à une condamnation absolue de l'imagination: l'extraordinaire puissance imaginative des personnages reste étroitement liée à l'idée de génialité mélancolique. Autour d'eux, c'est un univers fabuleux, plein de gaieté et soigneusement maîtrisé qui se met progressivement en place.

\section{Portrait du lecteur extravagant en fou lettré: l'émergence d'une folie livresque}

Le terme latin stultitia présente une ambiguïté sémantique: ainsi, la formule "stultitia loquitur", que l'on trouve au seuil de L'Éloge de la Folie d'Érasme, est communément traduite par "la Folie parle", mais le substantif pourrait également être rendu par le terme de sottise (Céard, 2004: 25). La notion d'extravagance n'est pas synonyme de sottise, si l'on entend ce vocable au sens de niaiserie, d'absence d'esprit, de bêtise. À l'inverse de l'insipiens ou du de-mens, qui sont des mots construits au moyen de préfixes privatifs, l'extravagant ne se caractérise pas par une absence ou par un manque de jugement. Lysis, le "berger extravagant" de Sorel, est un jeune garçon à l'esprit fin ${ }^{3}$, qui a étudié: après la mort de son père, un riche marchand de soie, son tuteur et cousin Adrian lui fait faire de coûteuses études au collège de Navarre. Il le presse par la suite d'entreprendre une carrière juridique, afin d'acquérir une charge de conseiller, mais le héros délaisse très vite les livres de droit pour ne plus lire que des romans, au grand désespoir d'Adrian. Celui-ci a beau lui confisquer ses livres, et même les brûler - souvenir de l'autodafé de la bibliothèque de Don Quichotte -, le jeune homme en rachète secrètement et en cache sous son lit ou dans ses chausses. II s'enferme dans sa chambre, ne mange qu'une fois par jour et passe son temps à lire ou à se réciter des petites bergeries tirées de ses lectures: six années de ce régime de vie (entre 18 et 24 ans) achèvent de le plonger dans la folie et l'amènent à quitter la demeure de son cousin afin de gagner, sous le pseudonyme de Lysis, la campagne de Saint-Cloud, où il espère pouvoir voir et vénérer celle qu'il appelle Charite, qui n'est en fait qu'une humble servante. Comme les personnages de L'Astrée, paradigme des romans pastoraux qu'il prétend imiter, Lysis est donc un berger lettré, qui s'essaie, avec plus ou moins de maladresse, à la composition poétique et musicale. D'où l'incompréhension comique qui l'oppose au paysan rustique qu'il rencontre au début du premier livre de l'œuvre:

\footnotetext{
${ }^{3}$ Dans les "Remarques" qui accompagnent le dixième livre du Berger, Sorel met en valeur la double part de finesse et de folie qui habite l'esprit du héros: "L'esprit de Lysis s'est tousjours faict paroistre subtil parmy ses plus grandes extravagances" (Sorel, 1628: 461).
} 
Bien que ce fust un gros rustique, \& qu'il luy vist des habits qui estoient fort differens des siens, il ne laissa pas de l'acoster avec un geste aussi courtois, que si c'eust esté Celadon ou Silvandre. Gentil berger, luy dit-il, apren moy quelles sont icy tes occupations. Songes-tu aux rigueurs de Clorinde? Combien y a t'il que tu n'as fait de chanson pour elle? Monstre moy de tes vers je te prie.

Ce Berger qui n'entendoit non plus ces mignardises que s'il luy eust parlé en langage barbare, s'estonna beaucoup de sa façon, ne sçachant quel homme c'estoit. Toutefois comprenant son discours le mieux qu'il luy estoit possible, il luy respondit, Je ne sçay pas ce que vous me voulez dire de Coq d'Inde; pour une chanson, j'en achetay l'autre jour une à Paris, au bout du Pont-neuf, \& pour des vers si ce sont des vers de terre que vous me demandez, j'en ay chez nous plein le cu d'une bouteille: ils me servent à pescher à la ligne, quand je me veux recréer (Sorel, 1627: 40-41).

Le rustre, qui n'a pas étudié, et pour qui la terre est un gagne-pain, non un décor bucolique susceptible d'accueillir des intrigues amoureuses, des chansons et des danses, se révèle incapable de comprendre les métaphores poétiques que lui débite Lysis. Le "berger extravagant", en prenant au pied de la lettre les images hyperboliques ressassées par les poètes galants, introduit entre les hommes de nouvelles formes de dissensions linguistiques post-babéliennes, qui rendent toute communication impossible. S'ensuit un dialogue fondé sur le quiproquo comique, au cours duquel Lysis, en décrivant Charite comme la grande prêtresse de l'amour, la fait passer aux yeux du paysan pour une sorcière aux pouvoirs terrifiants, dont il serait lui-même l'époux, sous les traits de l'Antéchrist. Une fois rentré au village, le rustre prévient ses compagnons de l'imminence de la fin du monde: les villageois de Saint-Cloud, terrorisés, passent la nuit à s'enivrer, avant de tomber dans la confusion, le lendemain matin, lorsque la folie du berger leur est révélée. Ainsi, le savoir sans maîtrise du lecteur extravagant, incapable de déjouer les excès et les mensonges de la parole fabuleuse, croise l'ignorance naïve et fruste des paysans, dépourvus de toute connaissance livresque. Dans les "Remarques" que Sorel publie en 1628 dans le deuxième volume du Berger, en vue d'éclairer et d'expliciter chacun des livres de I'histoire de Lysis, l'auteur précise par ailleurs que le savoir de son berger ne se limite pas aux romans pastoraux: il "n'a pas seulement leu les Romans, mais tous les livres aussi où il y avoit que[I]que chose qui pust convenir a son humeur" (Sorel, 1628: 549).

L'esprit de Carmelin, qui devient le valet du berger à partir du quatrième livre, n'est pas davantage réductible au bon sens populaire de ces paysans. Ayant effectué plusieurs professions et servi différents maîtres avant de rencontrer Lysis, il apparaît comme un pédant capable des réparties les plus fines comme des remarques les plus naïves et grossières: avant tout soucieux de son confort et de la satiété de son estomac, il accepte de rester auprès du berger, malgré les extravagances de celui-ci, pour profiter avec lui de la 
bonne chère que leur offrent Anselme, Clarimond, Hircan et les autres seigneurs présents en Brie. Les "Remarques" qui portent sur ce personnage témoignent tantôt de ses capacités réflexives, tantôt de son ignorance rustique. Sorel assure que "les femmes \& les hommes qui n'ont pas estudié ne connoissent pas la grace des discours de Carmelin" (Sorel, 1628: 191), mais il laisse aussi planer le doute sur la capacité de son jugement à fonctionner raisonnablement. Ainsi, lorsque Lysis s'apprête à partir avec lui vers une île exotique afin de délivrer Panphilie, - dans une mise en scène inventée par Hircan et ses amis -, Carmelin s'inquiète de savoir si les moutons qui ne vivent pas en France parlent une langue étrangère:

L'on ne sçayt si Carmelin avoit fait sa demande par malice où par naïveté: C'est une chose dont l'on n'a pû estre esclaircy à cause de l'inegalité de son esprit qui passoit quelquefois de la subtilité à la niayserie (Sorel, 1627: 303-304).

Le couple formé par Don Quichotte et Sancho Pansa est donc retravaillé autour d'une opposition qui ne permet plus de trancher entre la folie livresque et l'absence de lettres: tandis que Sancho était un paysan rustre et analphabète, qui suivait son maître, au moins dans la première partie de l'œuvre, en lui vouant une confiance naïve, Carmelin appartient quant à lui au type comique du pédant qui a étudié, mais qui, au contact de mauvais maîtres (l'hypocondriaque Lancelot, un Docteur, un faiseur d'almanachs, etc.) et d'œuvres de piètre valeur, n'est devenu capable que de répéter sans les comprendre des fragments embrouillés des textes lus. L'extravagance, en tant que folie liée au savoir, se sépare donc en deux ramifications: d'une part, Lysis, Don Clarazel, Juliette d'Arviane, dont l'entendement est capable des plus fines gentillesses de l'esprit, mais qui se sont égarés dans des lectures romanesques nuisibles; d'autre part, Carmelin, ou encore Ragotin dans Le Roman comique de Scarron ${ }^{4}$, chez qui le savoir ne donne lieu qu'à un psittacisme stérile de paroles non comprises et galimatiesques.

On voit donc que la notion d'extravagance est bien loin d'être systématiquement synonyme de folie. Là encore, deux types de personnages se distinguent: d'une part, ceux qui relèvent de ce que l'on peut nommer une folie-analogie; d'autre part, ceux dont l'égarement est présenté comme une authentique folie-pathologie. Dès son apparition dans Le Roman comique, Ragotin, décrit comme "un petit homme veuf, avocat de profession, qui avait une petite charge dans une petite juridiction voisine" (Scarron, 1985: 59), est parodiquement assimilé au personnage éponyme du Roland furieux de l'Arioste ${ }^{5}$. Bien qu'il permette d'introduire dans le récit la première nouvelle enchâssée, "L'Histoire de l'amante

\footnotetext{
4 "Il avait étudié toute sa vie; et, quoique l'étude aille à la connaissance de la vérité, il était menteur comme un valet, présomptueux et opiniâtre comme un pédant et assez mauvais poète pour être étouffé s'il y avait de la police dans le royaume" (Scarron, 1985: 59).

5 "C'était le plus grand petit fou qui ait couru les champs depuis Roland" (ibid.).
} 
invisible", traduite et adaptée d'une novela de Castillo Solorzano, ce pédant se caractérise par ses goûts littéraires archaïques, détachés de toute prise en compte des notions de bienséance et de vraisemblance: à la manière d'Alexandre Hardy, il est l'auteur d'une pièce intitulée Les Faits et les gestes de Charlemagne, en vingt-quatre journées, et il entend transformer la nouvelle qu'il vient de lire en comédie, aux dépens des réalités scéniques les plus élémentaires. Comme lui, le poète Roquebrune, autre personnage de pédant, fait figure de mauvais auteur et, de façon complémentaire, de mauvais lecteur. Lorsque le conseiller au parlement de Rennes nommé La Garouffière défend devant les comédiens le modèle de la novela espagnole, en citant l'exemple des Nouvelles exemplaires de Cervantès, Roquebrune lui oppose l'idéal des romans fleuves fabuleux:

[...] Roquebrune ne fut pas de cet avis. II dit fort absolument qu'il n'y avait point de plaisir à lire des romans s'ils n'étaient composés d'aventures de princes, et encore de grands princes, et que par cette raison-là l'Astrée ne lui avait plu qu'en quelques endroits. Et dans quelles histoires trouverait-on assez de rois et d'empereurs pour vous faire des romans nouveaux? Lui repartit le conseiller. II en faudrait faire, dit Roquebrune, comme dans les romans tout à fait fabuleux et qui n'ont aucun fondement dans l'histoire. Je vois bien, repartit le conseiller, que le livre de don Quichotte n'est pas trop bien avec vous. C'est le plus sot livre que j'aie jamais vu, reprit Roquebrune, quoiqu'il plaise à quantité de gens d'esprit. Prenez garde, dit le Destin, qu'il ne vous déplaise par votre faute plutôt que par la sienne (Scarron, 1985: 166).

Lorsqu'il vise Ragotin et Roquebrune, l'adjectif fou est employé au sein d'expressions à valeur hyperbolique, afin de discréditer des jugements esthétiques et littéraires jugés impertinents et intenables. Mais cette folie-analogie peut aussi être érigée en folie-pathologie chez d'autres personnages de mauvais lecteurs, tels Lysis, Don Clarazel, ou encore "la fausse Clélie": conformément à la théorie des quatre humeurs héritée d'Hippocrate et de Galien, qui associe à chaque individu un tempérament naturel dominé par le sang, la bile jaune, le flegme ou la bile noire, le trouble qui s'empare de leur esprit est cette fois-ci présenté comme une véritable forme de mélancolie. L'excès de bile noire, associé aux effets produits par les romans sur leur jugement, constitue l'étiologie de leur folie. Don Clarazel, dont le tempérament se définit comme sanguin et colérique, du fait de sa jeunesse, de sa fougue et de ses origines espagnoles, n'est pas mélancolique par nature. Mais, dès l'ouverture du récit, il se met à souffrir d'une passion désespérée à l'égard de sa maîtresse Sylviane, qui l'a rejeté: décidé à fuir toute compagnie, il se réfugie dans sa chambre, où il laisse libre cours à ses rêveries les plus alambiquées. L'enfermement, le manque d'activité physique et de divertissement, l'oisiveté, alliés au manque de sommeil et de nourriture, vont 
en l'espace de trois semaines le faire basculer dans une mélancolie dite aduste, ou encore contre-nature, par cuisson excessive des humeurs naturellement dominantes dans sa physiologie: cette cuisson produit en effet des vapeurs de bile noire qui, à partir des hypocondres - il s'agit d'un "chevalier hypocondriaque" -, vont progressivement obscurcir son cerveau. Toutefois, c'est la lecture assidue des romans d'Amadis qui, en trois semaines supplémentaires, aboutit à imprimer dans son jugement l'illusion que le monde des chevaliers errants correspond au monde dans lequel il vit. Lysis est lui aussi décrit comme un atrabilaire, submergé par la bile noire à la suite de plusieurs années de jeûnes, de veilles, d'oisiveté, de rêveries stériles, et surtout, de lectures de bergeries et de fictions sentimentales. Sorel sème soigneusement, tout au long des quatorze livres du Berger, des indices qui nous permettent de diagnostiquer chez le héros un cas précis de mélancolie:

Ses cheveux estoient un peu plus blonds que roux, mais frisez naturellement en tant d'anneaux qu'ils monstroient la seicheresse de sa teste, \& son visage avoit quelques traits qui l'eussent fait paroistre assez agreable, si son nez pointu \& ses yeux gris à demy retournez \& tout enfoncez ne l'eussent rendu affreux, monstrant à ceux qui s'entendoient à la Physionomie, que sa cervelle n'estoit pas des mieux faites (Sorel, 1627: 3-4).

Comme l'a montré Martine Alet (Alet, 2002), ce portrait physique du héros révèle, de la même manière que pour Clarazel, un tempérament dominé à la fois par la bile jaune et le sang. C'est donc la cuisson excessive (ou adustion) de ces deux humeurs qui aboutit à faire basculer le héros dans la mélancolie. L'étiologie de la folie de Lysis et de Clarazel montre à quel point les auteurs d'histoire comique puisent dans le savoir médical du temps pour fonder en légitimité, mais aussi asseoir sur des autorités savantes, le portrait du lecteur extravagant.

Ainsi, les deux personnages, au moment de leur guérison, feront l'objet d'un double traitement somatique et moral: dans le dernier chapitre du Chevalier hypocondriaque, Clarazel est soigné par Sylviane, qui, regrettant de s'être montrée si cruelle envers lui, utilise l'ascendant psychologique qu'elle détient sur son esprit pour le forcer à dire, et même à penser, que les romans de chevalerie ne sont que des fables mensongères; elle lui donne par la suite des bains parfumés à l'ellébore, plante reconnue depuis l'Antiquité comme un puissant purgatif. Quinze jours de ce régime suffisent à guérir définitivement le chevalier de ses erreurs passées. Chez Lysis, c'est avant tout le traitement moral, entrepris et dirigé par Clarimond dans le dernier livre de l'œuvre, qui l'amène à reconnaître que les bergers des pastorales qu'il a lues ne sont que des personnages fictionnels. Cette suprématie de la dimension psychologique de la maladie livresque face à sa réalité somatique montre donc 
que cette folie-pathologie doit avant tout être comprise comme une forme hyperbolique de la folie-analogie étudiée précédemment. En attribuant aux romans fabuleux le pouvoir nocif de plonger l'esprit de leur lecteur dans la mélancolie, Du Verdier et Sorel cherchent avant tout à renforcer le pouvoir critique de la satire qu'ils effectuent de ces livres. Contrairement à ces fictions pernicieuses, leurs ouvrages seront dotés d'une visée prophylactique: celle d'empêcher leur propre lecteur de succomber à la folie livresque.

Selon Patrick Dandrey, "dans l'Europe de la fin du XVI siècle et du début du XVII", il n'est pas exagéré de dire qu'être mélancolique fut à la mode" (Dandrey, 2003: 17): que ce soit en Espagne, en Angleterre, en France, comme dans le reste de l'Europe, à la Renaissance et à l'âge classique, l'humeur noire domine les arts iconographiques et littéraires, au travers de personnages comme Don Quichotte, Hamlet, ou le Misanthrope de Molière. En liant l'extravagance livresque à cette vogue mélancolique, les auteurs d'histoires comiques placent donc consciemment leurs personnages au sein d'un imaginaire culturel commun.

\section{II. “Qu'ils lisent eux mesmes, \& qu'ils jugent, \& puis l'on verra s'ils ont un bon sens" (Sorel, 1628: 672-673): le lecteur extravagant comme contre-modèle de narrataire.}

Dans les quatorze livres de "Remarques" qui accompagnent le Berger, le mot extravagance est régulièrement associé aux fictions sentimentales et pastorales ${ }^{6}$. Entendu au sens d'excès dès le milieu du $X V l^{e}$ siècle, le substantif vient sanctionner un type de roman accusé de recourir sans mesure à l'imaginaire le plus débridé. Sorel s'en prend avant tout aux romans pastoraux qui, dans le sillage de l'Astrée, emportent les suffrages des lecteurs, et surtout des lectrices, au cours des trente premières années du siècle ${ }^{7}$. Mais, en leur donnant pour parenté directe la poésie fabuleuse et les textes mythologiques de l'Antiquité - telles les œuvres d'Homère, de Virgile et d'Ovide - l'auteur du Berger montre qu'il vise plus largement un type de fiction fondé sur l'invraisemblance et le merveilleux. Le treizième livre de l'histoire de Lysis est tout entier consacré au procès pro et contra du genre romanesque, dans un épisode qui sert de préliminaire à la guérison du héros. Dans un premier temps, Clarimond se charge du réquisitoire: passant en revue les fictions poétiques et fabuleuses les plus anciennes, telles L'lliade et L'Odyssée, L'Énéide, Les Métamorphoses d'Ovide, ou Les Éthiopiques d'Héliodore, de même que des textes d'auteurs plus récents,

\footnotetext{
6 “Puisque j'ay dit ce que c'est qu'un Roman, l'on connoist quels ouvrages je veux blasmer: mais l'on voudroit possible sçavoir encore, si outre les inventions extravagantes qui sont dans les Romans \& dans la poësie, je ne veux point aussi condamner la rime, \& l'art de faire des vers [...]" (Sorel, 1628: 12).

${ }^{7}$ Sur l'histoire du genre romanesque au XVII siècle, voir, entre autres, les ouvrages de Maurice Lever et Camille Esmein-Sarrazin cités en bibliographie.
} 
comme L'Arioste, le Tasse, ou Ronsard, sans oublier L'Astrée, le personnage, auquel Sorel donnera largement raison dans ses "Remarques", condamne l'impiété de ces œuvres qui détournent leurs lecteurs de la foi chrétienne, mais aussi de la morale. En mettant en scène des personnages qui cultivent sans frein la passion amoureuse, ils donnent de mauvais exemples aux jeunes gens et risquent de divertir les femmes du soin de leur honneur. Clarimond relève le caractère invraisemblable de ces héros parfaits, qui se rencontrent toujours à point nommé, dont l'identité royale est découverte au moment où ils s'apprêtent à épouser la plus belle des princesses, et dont les aventures se répètent ad libitum selon une mécanique ridicule et lassante. Ce double manquement aux bienséances et à la vraisemblance forme la base de toutes les impertinences qu'il reproche aux romans:

Apres tant d'impertinences que j'ay trouvees dans les Romans \& dans la Poësie, vous voyez mon juge, que ce n'est pas sans sujet que je les mesprise, \& je vous diray bien que quand il y auroit un de ces ouvrages qui seroit exempt de toutes les fautes que j'ay remarquees, je ne le priserois pas tant que le moindre recit veritable qui se puisse trouver au monde. Vous aprenez dans une histoire des choses que vous pouvez alleguer pour des authoritez, mais dans un Roman il n'y à aucun fruict à recueillir. Au contraire la pluspart des esprits s'y perdent comme j'en donneray bien des tesmoignages sans sortir de cette compagnie (Sorel, 1628: 95-96).

Chez Sorel, le roman perd tout particulièrement la partie lorsqu'il est confronté aux livres d'histoire, genre dont le fondement s'appuie sur la vérité. Musardan, piètre auteur dont Lysis a lu les œuvres et qui assiste lui aussi à ce procès, au lieu de défendre son genre de prédilection, afin de remplir le rôle qu'on lui a octroyé, donne raison aux critiques de Clarimond en reconnaissant que ses propres livres sont pleins d'ornements mensongers. II ne possède ni les capacités rhétoriques, ni les connaissances livresques nécessaires pour répondre à celui-ci. Par ailleurs, le personnage du berger est là pour témoigner par sa personne même que ces textes tendent un miroir aux alouettes au lecteur: en faisant fi de toute chronologie, en mêlant indistinctement les mœurs et les coutumes, les territoires proches et lointains, mais aussi les religions païennes et chrétiennes, le roman se constitue en genre fondamentalement trompeur et pernicieux.

C'est en définitive Philiris qui prend en charge le plaidoyer des œuvres incriminées: reprenant point par point les critiques de Clarimond, ce seigneur ami d'Hircan, qui s'est luimême déguisé en berger et a promis à Lysis d'écrire le récit de ses aventures, relativise les récriminations de celui-ci en replaçant chaque livre dans son contexte historique et géographique; il fait avant tout du roman une lecture agréable, conçue pour le divertissement. Les fictions dont a parlé Clarimond sont des ouvrages "faits à plaisir", non 
soumis aux "fascheuses loix de l'histoire", dans lesquels on "peut mettre tout ce que l'on veut, tellement que l'on y void tous les exemples de vertu que l'on se peut imaginer" (Sorel, 1628: 149-150). II n'est ni honteux ni blâmable de prendre plaisir à la lecture de ces livres pleins de douceur et de "discours charmants" ${ }^{8}$. Alors qu'Anselme, désigné comme le juge de ce procès, hésite à trancher entre ces deux plaidoiries, Amarylle, épouse d'Hircan, demande à prendre la parole au nom des femmes lectrices de roman: c'est grâce à ce type de fictions que celles-ci, qui n'ont pas de précepteurs, peuvent être formées à l'école du monde. Mais c'est aussi par l'intermédiaire des romans que leurs amants et maris comprennent les subtilités de l'amour, qui ne se trouvent pas dans les livres de philosophie et de morale ${ }^{9}$. Le jugement final d'Anselme fixe donc la place du roman, lecture de plaisir qui s'inscrit dans le cadre d'un divertissement:

[...] nous ordonnons que puisque tous ces ouvrages fabuleux ne sont faits que pour donner du plaisir, \& que le dessein des Escrivains reussit assez bien quand ils peuvent recreer les lecteurs, il sera tousjours permis au peuple de chercher son contentement dans tous les livres où il le pourra treuver; \& d'autant que Clarimond à blasmé des livres qui ne meritent pas de l'estre tant, \& que Philiris en a loüé aussi qui ne sont pas dignes de ses loüanges, les bons esprits aviseront par cy apres à juger sans passion des divers ouvrages qui se presenteront (Sorel, 1628: 154-155).

Il est donc capital de se constituer une bonne bibliothèque dans laquelle chaque type de livre aura sa place et sa fonction soigneusement délimitées ${ }^{10}$. Lorsqu'il mène Lysis vers la guérison, à la fin du Berger, Clarimond veille à ce qu'il sélectionne des lectures profitables parmi les ouvrages qu'il possède dans son étude. Mais le berger menace de retomber dans l'extravagance, non plus cette fois-ci à cause des pastorales, mais à force de lire des ouvrages de philosophie morale! Clarimond doit alors de nouveau l'empêcher de "faire par tout le Philosophe Stoique ou le Philosophe Cynique" (Sorel, 1628: 246). L'extravagance des romans et de la poésie fabuleuse n'est donc pas la seule responsable du trouble de l'esprit

\footnotetext{
${ }^{8}$ À propos de L'Histoire des amours de Lysandre et de Caliste de Vital d'Audiguier, Philiris décrit ainsi le plaisir ressenti par le lecteur: "C'est là \& en beaucoup d'autres lieux qu'il se trouve des discours si charmans que bien que l'on en desire vistement sçavoir la fin, l'on voudroit neantmoins que le livre ne finist jamais" (Sorel, 1628: 146).

9 "Nous autres femmes qui n'allons point au college, \& qui n'avons point de precepteurs comme les hommes pour nous aprendre les diverses choses qui se passent au monde, c'est seulement dans les Romans que nous avons le moyen de nous rendre sçavantes. Si l'on nous les oste lon nous rendra toutes stupides \& toutes sauvages: car nos esprits n'estans pas propres aux livres de philosophie n'y aux autres ouvrages serieux, ce n'est pas la que nous pouvons aprendre ny la vertu ny l'Eloquence. Qui plus est l'on nous fera un grand tort, pource que nos amans \& nos marys ne s'adonnant plus aussi à cette agreable lecture, mettront en oubly toutes les gentillesses de l'amour, tellement que nous ne serons plus servies avecque passion, \& que nous n'aurons plus d'avantures qui donnent matiere d'escrire aux Autheurs du siecle. Songez à cela nostre juge, \& vous representez que si vous condamnez les Romans vous ne ferez pas seulement tort à toutes les femmes, mais aussi à tous les hommes qui ne les trouveront plus aymables comme autrefois" (Sorel, 1628: 152-153).

${ }^{10}$ En 1664, Sorel composera en ce sens une Bibliothèque françoise de M. C. Sorel, ou le Choix et l'examen des livres françois qui traitent de l'éloquence, de la philosophie, de la dévotion et de la conduite des mœurs.
} 
qui frappe Lysis: chez les lecteurs, on rencontre des cerveaux affaiblis qui sont plus susceptibles que d'autres de succomber à ce type de folie livresque. Les torts sont partagés: à l'impertinence des "mauvais livres", répond le mauvais jugement du lecteur extravagant.

Ainsi, les histoires comiques mettent en scène des personnages de mauvais lecteurs qui apparaissent comme des contre-exemples de ce que doit être le narrataire ${ }^{11}$, figure virtuelle du lecteur postulée par l'œuvre; plus précisément, ces lecteurs extravagants s'écartent de ce qu'Umberto Eco appelle le "Lecteur Modèle", ce "lecteur-type que le texte prévoit comme collaborateur, et qu'il essaie de créer" (Eco, 1996: 17) ${ }^{12}$. L'extravagant apparaît donc comme une figure de lecteur repoussoir: celui qui, sans distance critique, sans recul réflexif, se laisse entraîner au fil des mots et embarquer sans résistance sur une nef qui le mènera insidieusement aux confins du monde raisonnable, sur les terres de la folie. Le lecteur réel du Berger, du Chevalier, ou encore du Roman comique, se doit d'inverser la façon qu'ont Lysis, Clarazel, Ragotin ou Roquebrune de lire, afin de retrouver les traits caractéristiques du Lecteur Modèle. Par l'intermédiaire de ces figures extravagantes, I'histoire comique a donc pour ambition de refonder le pacte de lecture: si Sorel, Du Verdier et Subligny choisissent de mettre en scène des personnages devenus fous, afin de détourner le lecteur d'un tel rapport aux livres, Scarron, dans Le Roman comique, malmène celui-ci en entrecroisant le récit premier de récits seconds, parfois inachevés, en multipliant les narrateurs intradiégétiques, en intitulant les différents chapitres au moyen d'énoncés déceptifs $^{13}$. Jouant avec la figure de la métalepse ${ }^{14}$, l'auteur ne cesse de briser le cours de sa narration pour en démonter les rouages et les artifices. Mais il convoque également différentes figures de narrataires, tantôt sérieuses tantôt ridicules, tantôt "bénévoles" tantôt "malévoles"15, pour mieux disséminer les éléments constitutifs de son Lecteur Modèle. Le lecteur réel de l'œuvre ne peut donc pas s'abandonner aux rets séducteurs de l'imaginaire, comme les romans fabuleux l'invitent à le faire: il est au contraire pressé de réagir, de rester continuellement en éveil, de déchiffrer, d'interpréter, de faire sans cesse fonctionner son

\footnotetext{
${ }^{11}$ Sur le narrataire, voir Genette, 1972: 265-267.

12 Voir également Umberto Eco, Lector in fabula. Le rôle du lecteur ou la Coopération interprétative dans les textes narratifs (1985).

13 "Qui contient ce que vous verrez, si vous prenez la peine de le lire" (I ${ }^{\text {è }}$ partie, chap. XI); "Qui peut-être ne sera pas trouvé fort divertissant" (I ère partie, chap. XXI); "Des moins divertissants du présent volume" (II" partie, chap. XI).

${ }^{14}$ Gérard Genette définit la métalepse comme "[...] une manipulation - au moins figurale, mais parfois fictionnelle [...] - de cette relation causale particulière qui unit, dans un sens ou dans l'autre, l'auteur à son œuvre, ou plus largement le producteur d'une représentation à cette représentation elle-même" (Genette, 2004: 14). II y a métalepse lorsque l'auteur de l'œuvre, le lecteur ou l'un des personnages de celle-ci franchit les frontières du niveau de fictionnalité dans lequel il se trouve. Ainsi, le narrateur du Roman comique se met en scène à plusieurs reprises comme un auteur nonchalant et peu soucieux de l'œuvre qu'il est en train de produire: "Il accepta l'offre qu'elle lui fit, et, cependant que ses bêtes mangèrent, l'auteur se reposa quelque temps et se mit à songer à ce qu'il dirait dans le second chapitre" (Scarron, 1985: 39).

15 "Tandis que le bruit de tant de personnes, qui riaient ensemble, diminue peu à peu et se perd dans l'air, de la façon à peu près que fait la voix des échos, le chronologiste fidèle finira le présent chapitre sous le bon plaisir du lecteur bénévole ou malévole, ou tel que le ciel l'aura fait naître" (Scarron, 1985: 239).
} 
jugement. C'est cette dernière faculté que les histoires comiques sollicitent en premier lieu, au lieu de stimuler la puissance imaginative.

Les lecteurs extravagants et les bons lecteurs ne forment pas pour autant deux catégories hermétiquement séparées. Comme nous le montre le parcours du jeune Francion, dans L'Histoire comique de Francion de Sorel, l'enfance est un moment critique au cours duquel l'on est particulièrement susceptible de tomber dans le piège d'un recours sans brides au fabuleux:

C'estoit donc mon passe-temps que de lire des Chevaleries, et faut que je vous die que cela m'espoinçonnoit le courage, et me donnoit des desirs nompareils d'aller cercher les avantures par le monde. Car il me sembloit qu'il me seroit aussi facile de couper un homme d'un seul coup par la moitié, qu'une pomme. J'estois au souverain degré des contentements quand je voyois faire un chapelis horrible de Geans dechiquetez menu comme chair a pasté. Le sang qui issoit de leurs corps a grand randon faisoit un fleuve d'eau roze, où je me baignois moult delicieusement, et quelquesfois il me venoit en l'imagination que j'estois le mesme Damoisel qui baisoit une Gorgiase Infante qui avoit les yeux verds comme un Faulcon (Sorel, 1958: 174).

Le temps du collège marque pour Francion le moment de la découverte des romans de chevalerie et des fables amoureuses, ce qui menace de le transformer en jeune Don Quichotte. Mais la maturité acquise au fil des années le détournera de tels ouvrages et lui apportera la distance apte à lui permettre de jeter un regard amusé et critique sur ses lectures passées. L'adjectif extravagant et ses dérivés, bien qu'ils soient d'abord apparus au XIV ${ }^{e}$ siècle dans le lexique scolastique, au sens d'"en dehors des textes canoniques", font l'objet d'acceptions pleinement négatives dans les œuvres que nous avons mentionnées: ils désignent un mode d'écriture et de lecture déraisonnable et dangereux. Pourtant, les lecteurs fous que sont Lysis ou Clarazel, comme leur ancêtre Don Quichotte, sont loin d'être des personnages intégralement condamnés: l'extravagance suppose aussi un rapport à l'imagination qui peut se révéler subtil, voire admirable.

\section{De l'extravagance à l'extraordinaire: éloge de l'imagination}

Si Francion enfant s'est vu sur le point de basculer dans la folie livresque, danger dont il sait bien se garder une fois devenu adulte, c'est que le cerveau mou du jeune âge est particulièrement réceptif aux fausses impressions transmises par l'imagination. Conçue comme l'une des trois puissances de l'âme, la faculté imaginative transmet au jugement les représentations qu'elle tire du témoignage des cinq sens externes, ou bien qu'elle forme à 
partir d'objets absents. Le jugement conserve par la suite certaines de ces impressions dans la mémoire, qu'il est susceptible de convoquer à sa convenance ${ }^{16}$. Mais lorsque l'imagination est perturbée, notamment par les vapeurs de la bile noire, elle se fait trompeuse messagère et envoie de fausses impressions vers l'intellect, qui en conçoit des hallucinations et des chimères. La séparation des trois facultés de l'âme explique qu'elles puissent être affectées séparément par la maladie. Chez Lysis, Clarazel ou Juliette d'Arviane, seule l'imagination est touchée: le jugement et la mémoire continuent de bien fonctionner. Les maladies mélancoliques se caractérisent d'autre part par leur discontinuité: l'atrabilaire peut connaître des phases de lucidité, comme c'est le cas pour la "fausse Clélie", dont les accès durent généralement une douzaine d'heures, ou bien peut délirer sur un objet précis en particulier.

Dans le cas du lecteur extravagant, les fausses impressions que l'imagination transmet au jugement prennent appui sur les fictions fabuleuses. C'est parce qu'il croit vivre dans le Forez de Céladon et d'Astrée que Lysis prend Hircan, qui se promène avec un bâton, à la manière des seigneurs de la région de Brie, pour un puissant magicien (Sorel, 1627: 525). Mais, comme dans la seconde partie de Don Quichotte ${ }^{17}$, les personnages que le berger et Clarazel croisent sur leur chemin s'appliquent à rendre le monde conforme à leurs délires imaginatifs: Hircan fait effectivement semblant d'être doté de pouvoirs magiques; le comte d'Oran se fait passer auprès du "chevalier hypocondriaque" pour le chevalier de la Rose Verte et met en scène, avec le marquis d'Artigny, le combat du héros contre un monstre, qui est en fait un dogue déguisé, puis contre un chevalier rendu invincible par une magicienne, qui n'est qu'un gentilhomme actionné de bas en haut au moyen d'une escarpolette. Le monde n'est donc pas ontologiquement trompeur: ce sont les seigneurs et dames que les lecteurs extravagants rencontrent qui le rendent tel. Les personnages évoluent de ce fait dans un perpétuel décor de mascarade et de fête costumée, dans lequel ceux qui les observent se complaisent avec joie: c'est bien là le signe que le monde de l'imaginaire est source de divertissement et de gaieté, à condition qu'il reste maîtrisé.

II serait donc erroné de lire dans les histoires comiques du premier $\mathrm{XVII}{ }^{e}$ siècle une condamnation radicale de l'imagination. Le personnage de Don Quichotte témoignait déjà de l'influence exercée sur Cervantès par l'ouvrage du médecin Juan Huarte de San Juan intitulé Examen de Ingenios (1575). Celui-ci, à l'encontre de la traditionnelle condamnation thomiste de l'imagination, distingue trois catégories de tempérament naturel: à côté des hommes dominés par la mémoire, particulièrement doués pour les langues (dont le latin), la grammaire, la cosmographie, l'arithmétique, etc., on trouve ceux chez qui prédomine l'entendement et qui sont propres à la théorie de la médecine, à la dialectique, à la

\footnotetext{
${ }^{16}$ Sur l'imagination à l'âge classique, voir, entre autres, Gérard Ferreyrolles, Les Reines du monde: limagination et la coutume chez Pascal (1995), Pierre Ronzeaud (dir.), L'Imagination, Littératures classiques, $n^{\circ} 45$, printemps 2002, et Michèle Rosellini, "Le Berger extravagant, critique de l'imagination ou imagination critique?" (2002).

${ }_{17}$ Voir Fanlo, 2004: 33-41.
} 
philosophie naturelle et morale, à l'art de la plaidoirie, etc.; enfin, ceux dominés par l'imagination, susceptibles d'être poètes, musiciens, prédicateurs, peintres, aussi bien que gouverneurs, chefs militaires, ingénieurs, etc. C'est donc cette dernière puissance qui rend l'homme compétent dans le plus grand nombre de domaines. II appartiendra à chaque être de découvrir quelle est sa disposition propre et de cultiver uniquement les sciences pour lesquelles il est fait, sous peine de ne jamais réussir en rien. Dès le prologue de la première partie, Don Quichotte est décrit comme un "enfant sec, ratatiné, bizarre, plein de fantaisies diverses et jamais imaginées" (Cervantès, 2008: 127): le qualificatif d'ingenioso qui lui est attribué témoigne de la subtilité de certaines de ses pensées. Le mot fantaisie est également utilisé dans le sous-titre du Berger extravagant comme synonyme d'imagination: majoritairement envisagées dans un premier temps de manière péjorative (au sens de chimères, de visions de l'esprit), les saillies d'esprit du héros laissent de plus en plus la place, au fil des "Remarques", à des commentaires admiratifs de la part de l'auteur. Lysis a beau avoir le cerveau troublé, son entendement reste supérieur à celui des auteurs et des poètes qui ont composé les textes pernicieux qui l'ont rendu fou. II se révèle même capable à plusieurs reprises d'améliorer les œuvres dont il est féru, en trouvant de meilleures raisons que ceux qui les ont écrites pour en expliquer certains passages:

Toutefois j'admire l'imagination du berger qui asseure que le feu des yeux de Charite est invisible comme le feu elementaire, \& qu'il ne se fait voir que quand il a de la matiere à consumer. C'est en cela qu'il fait la leçon aux Poëtes \& aux autres Amans, qui jamais n'ont donné cette raison pour les feux de leurs maistresses qui ne se peuvent voir (Sorel, 1628: 103).

Le "berger extravagant" formule donc des subtilités d'esprit supérieures à celles des romanciers et des poètes, dont on a vu, avec Musardan, qu'ils cultivaient l'ignorance et le manque d'éloquence. Clarazel, quant à lui, est moins encore que Lysis présenté comme un antihéros ridicule: valorisé dès l'incipit du Chevalier par son tempérament sanguin et sa jeunesse valeureuse, le héros est décrit à plusieurs reprises comme un personnage doté de toutes les qualités physiques et faits pour inspirer de l'amour aux dames ${ }^{18}$. La vaillance héroïque qu'il montre au combat, au cours de ses aventures errantes, contribue également à rendre vraisemblable sa guérison définitive et totale, à la fin du roman, ainsi que son mariage avec la jeune et belle Sylviane. Cette vision partiellement positive des deux extravagants et de leur créativité imaginative s'appuie sur l'ambivalence de jugement porté depuis la plus

\footnotetext{
18 "[...] Plusieurs se moquerent de ses follies, quelques autres donnerent des larmes au malheur qui l'accompagnoit, \& tous generalement le louërent de deux choses, sçavoir est de la grace qu'il avoit à bien exprimer ce qu'il vouloit dire, \& de faire ses vaillances imaginaires avec un courage sans peur, de sorte qu'il passoit pour fort courageux dans l'estime des braves hommes, \& envers les femmes pour un cavalier tres-digne d'amour si son cerveau n'eust esté troublé par un malheureux accident" (Du Verdier, 1632: 150-151).
} 
haute Antiquité sur les mélancoliques: le célèbre Problème $X X X, 1$, attribué à Aristote, constate à quel point les hommes exceptionnels, qu'ils soient artistes, grands intellectuels ou fins politiciens, sont très souvent des êtres dominés par les vapeurs de la bile noire. Comme le vin, celles-ci sont susceptibles d'aiguillonner les facultés de l'esprit aussi bien que de les assoupir. Le mélancolique, selon le degré d'humeur noire qui l'excède, qu'il soit modéré ou au contraire incontrôlé, oscillera dangereusement entre les illuminations de l'homme de génie et les accès dévastateurs du furieux ${ }^{19}$. Les fantaisies de Lysis et Clarazel évoluent pareillement sur une ligne qui les fait basculer tantôt dans la folie la plus risible, tantôt dans l'inventivité la plus admirable: l'extravagance peut ainsi céder la place à l'extraordinaire.

C'est la raison pour laquelle les personnages qui croisent le chemin des lecteurs extravagants souhaitent tant les garder auprès d'eux et se divertir de leurs folies. Comme le faisaient déjà le duc et la duchesse que rencontrent Don Quichotte et Sancho dans la seconde partie de l'œuvre de Cervantès, Anselme, Hircan et ses amis, dans le Berger, le baron d'Oran, le marquis d'Artigny, puis le baron de la Tour et leur entourage, dans le Chevalier, recréent un monde fabuleux et artificiel, dans lequel tout est mise en scène et où seuls les deux héros croient véritablement être le rôle qu'ils jouent. À propos du second volume de Don Quichotte, Jean-Raymond Fanlo a pu parler de "fête de l'imagination" et du roman (Fanlo, 2004: 33-41): les compagnons de Lysis et Clarazel apportent la preuve du plaisir que l'on peut prendre en se plongeant - en toute connaissance de cause - dans l'univers des romans. Léonor, Angélique et Oronte, lorsqu'ils accueillent Lysis prétendument déguisé en fille, se pressent même de relire des fictions pastorales afin d'adapter leur conduite et leurs propos à cette nouvelle fantaisie du berger ${ }^{20}$. Afin de parodier la mode des récits insérés dont les grands romans fabuleux usent et abusent, Sorel conduit plusieurs de ses personnages à faire le récit imaginaire et fantaisiste de leurs aventures passées. Au début du huitième livre, Philiris, Fontenay, Polidor et Méliante, travestis en bergers, content leur histoire devant Lysis et l'ensemble des personnages réunis en assemblée: "Ils ne dirent rien que de tres-agreable, soit qu'il y eust du mensonge ou de la verité" (Sorel, 1627: 169). Peu importe que leurs récits soient des fables, du moment que tous, à l'exception du "berger extravagant", aient conscience que les orateurs manipulent et parodient les ressorts des narrations imaginaires. De même, la compagnie met en scène deux pièces de théâtre à sujet mythologique, qui traitent du ravissement de Proserpine par Pluton et de la conquête de la Toison d'Or, et dans lesquelles chaque personnage s'exprime selon un style spécifique (hyperbolique, métaphorique, galimatiesque, etc.). Cette étrangeté linguistique permet de

\footnotetext{
${ }^{19}$ Cette forme de survivance du Problème $X X X, 1$ va donc dans le sens contraire de l'évolution générale que Marc Fumaroli constate à partir de la fin de la Renaissance, époque qui marque selon lui la fin du prestige attaché à la mélancolie (Fumaroli, 1994: 403-439).

20 "Les entretiens de Lysis avoient obligé Oronte, Floride, Leonor, \& Angelique à lire des Romans pour se rendre sçavans en sa doctrine, \& tirer plus de plaisir de luy" (Sorel, 1627: 549).
} 
maintenir le jeu à distance et donne aux fables antiques une portée fortement comique: une fois encore, seul Lysis se laisse pleinement happer par son rôle et ressort de cet épisode plus fou que jamais (Sorel, 1627: 351 ss).

Il existe donc une forme de recours mesuré à l'imaginaire et à l'univers fictionnel, qu'il convient de privilégier. C'est cette conception maîtrisée du romanesque, "selon la portée de I'humanité" (Scarron, 1985: 166), que Scarron propose au lecteur dans Le Roman comique, lorsqu'il insère quatre nouvelles traduites et adaptées de novelas espagnoles, de même que des récits seconds pris en charge par des narrateurs intradiégétiques. L'ensemble de ces narrations enchâssées ne se prive pas de recourir à certains des épisodes types des romans fabuleux (scènes d'innamoramento, enlèvements, fausses morts, reconnaissances, etc.); mais les héros mis en scène ne sont plus des êtres parfaits, qui ne connaissent ni la peur, ni la fatigue, ni le doute, et qui n'écoutent jamais les besoins de leur corps, les lieux de l'action ne sont plus des espaces exotiques et fantasmés, la chronologie reste proche de celle du lecteur, tandis que le souci de la vraisemblance demeure omniprésent. C'est donc une forme de "romanesque hybride" que met en place Scarron, qui ne renonce jamais à captiver l'esprit de son lecteur, mais reste toujours tempéré par la raison. Le plaisir de la lecture est toujours prédominant: mais celui qui tient l'ouvrage entre ses mains ne court plus aucun risque de se laisser emporter jusqu'à devenir extravagant.

\section{Conclusion}

Il y en a qui ayment ce livre, \& qui ne seroient pas bien ayses que Clarimond en dist davantage: mais ils ne considerent pas, que si cela estoit, Philiris luy donneroit aussi plus de loüanges qu'il ne fait, \& qu'il respondroit à plusieurs objections que le peuple fait d'un costé \& d'autre, lesquelles demeureront indecises (Sorel, 1628: 692).

Le livre dont il est ici question n'est autre que L'Astrée, modèle par excellence des romans pastoraux, mais dont Sorel ne peut s'empêcher, par petites touches disséminées dans ses "Remarques", de reconnaître les mérites, conseillant même à son lecteur de s'y reporter $^{21}$. La vogue des lecteurs extravagants que nous avons pu décrire au sein de I'histoire comique révèle par conséquent que, si celle-ci se définit avant tout comme une sous-catégorie romanesque éminemment critique, qui entend écrire contre, la condamnation des romans fabuleux qu'elle accueille n'est pas diamétralement tranchée. L'extravagance, perturbation érudite et livresque de l'esprit, est susceptible de produire aussi bien les

\footnotetext{
21 "Les differends d'entre Laonice \& Tircis, \& plusieurs autres qu'il allegue, sont connus de tout le monde, aussi sont toutes les coustumes des bergers de Forests dont il parle. Que s'il se trouve quelqu'un qui ne les sçache point, il faut plustost qu'il les aille lire en leur place, que d'attendre que je luy raconte des choses si longues" (Sorel, 1628: 82-83).
} 
fantaisies les plus comiques que les imaginations les plus fines et subtiles: elle a pour pendant l'exceptionnel et l'extraordinaire.

C'est en partie ce rapport complexe et ambigu aux œuvres que le roman comique prend pour cibles qui suscitera sa progressive disparition, parallèlement à celle de la mode des grandes sommes romanesques, à partir de la fin des années 1660 . Un autre modèle de fiction s'imposera avec l'émergence de la nouvelle historique et galante. II n'en demeure pas moins que le personnage du lecteur extravagant ouvrira la voie à une longue réflexion sur le narrataire et les enjeux de la lecture, dont bénéficieront, au siècle suivant, Marivaux, Diderot, Laurence Sterne, et même, plus près de nous, Calvino et Echenoz. 


\section{Bibliographie}

Sources primaires:

Cervantes, Miguel de (1605-1615; 2008). Don Quichotte de la Manche. Paris: éd. et trad. de JeanRaymond Fanlo, Le Livre de Poche, coll. "La Pochothèque".

Du VERDIER, Gilbert Saulnier (1632). Le Chevalier hypocondriaque. Paris: Pierre Billaine.

SCARRON, Paul (1651-1657; 1985). Le Roman comique. Paris: éd. Jean Serroy, Paris: Gallimard, coll. "Folio classique".

SOREL, Charles (1623-1626-1633; 1958). Histoire comique de Francion. In: Romanciers du XVII siècle. Paris: éd. Antoine Adam, Paris: Gallimard, coll. "Bibliothèque de la Pléiade".

SOREL, Charles (1627). Le Berger extravagant, ou parmy des fantaisies amoureuses on void les impertinences des Romans \& de Poësie. Paris: Toussaint du Bray.

SOREL, Charles (1628). Le Berger extravagant, ou parmy des fantaisies amoureuses l'on void les impertinences des Romans \& de la Poësie. Troisiesme partie. Suivi des Remarques sur les XIIII livres du Berger Extravagant. Paris: Toussaint du Bray.

\section{Sources secondaires:}

ALET, Martine (2002). "Étude psycho-physiologique du Berger extravagant de Charles Sorel: la mélancolie de Louys". In: PFSCL, vol. XXIX, n56, pp. 153-175.

BARDON, Maurice (1931). Don Quichotte en France au XVII' et au XVIII siècles: 1605-1815. Paris: H. Champion.

CEARD, Jean (2004). "La sottise, la stupidité dans les Adages d'Érasme". In: Nicole Jacques-Lefèvre et Anne.-Pascale Pouey-Mounou (éds.), Sottise et ineptie, de la Renaissance aux Lumières: discours du savoir et représentations romanesques. Nanterre: Université Paris X-Nanterre, pp. 19-32.

DANDREY, Patrick (2003). Les Tréteaux de Saturne: scènes de la mélancolie à l'époque baroque. Paris: Klincksieck, coll. "Le Génie de la mélancolie".

Eco, Umberto (1985). Lector in fabula. Le rôle du lecteur ou la Coopération interprétative dans les textes narratifs. Paris: Grasset.

ECO, Umberto (1996). Six promenades dans les bois du roman et d'ailleurs. Paris: Grasset.

ESMEIN-SARRAZIN, Camille (2008). L'Essor du roman. Discours théorique et constitution d'un genre littéraire au XVII siècle. Paris: H. Champion.

FANLO, Jean-Raymond (2004). "La continuation du Quichotte ou les fêtes de l'imagination". In: Huguette Krief et Sylvie Requemora (éds.), Fête et imagination dans la littérature du XVI au XVIII siècle. Aix-en-Provence: Publications de l'Université de Provence, pp. 33-41.

FERREYROLLES, Gérard (1995). Les Reines du monde: l'imagination et la coutume chez Pascal. Paris: H. Champion.

FUMAROLI, Marc (1994). "La mélancolie et ses remèdes. Classicisme français et maladie de l'âme". In: La Diplomatie de l'esprit: de Montaigne à La Fontaine. Paris: Hermann, pp. 403-439.

GENETTE, Gérard (1972). Figures III. Paris: Seuil.

GENETTE, Gérard (2004). Métalepse. Paris: Seuil.

LeVER, Maurice (1996). Romanciers du Grand Siècle. Paris: Fayard.

RONZEAUD, Pierre (2002). L'Imagination, Littératures classiques, $\mathrm{n}^{\circ} 45$.

ROSELLINI, Michèle (2002). "Le Berger extravagant, critique de l'imagination ou imagination critique?". In: L'Imagination, Littératures classiques, $\mathrm{n}^{\circ} 45$, pp. 179-205.

Sermain, Jean-Paul (1999). Le Singe de don Quichotte. Marivaux, Cervantès et le roman postcritique. Oxford: Voltaire Foundation, coll. "Studies on Voltaire and the Eighteenth Century". 
SeRMAIN, Jean-Paul (2002). Métafictions (1670-1730). La réflexivité dans la littérature d'imagination. Paris: H. Champion.

SERROY, Jean (1981). Roman et réalité: les histoires comiques au XVII siècle. Paris: Minard. 